\title{
The role of miR-106p-5p in cervical cancer: from expression to molecular mechanism
}

\author{
Yuexiong $\mathrm{Yi}^{1}$, Yanyan Liu', Wanrong $\mathrm{Wu}^{1}$, Kejia $\mathrm{Wu}^{1}$ and Wei Zhang ${ }^{1}$
}

\begin{abstract}
This study aims to investigate the role of miR-106b-5p in cervical cancer by performing a comprehensive analysis on its expression and identifying its putative molecular targets and pathways based on The Cancer Genome Atlas (TCGA) dataset, Gene Expression Omnibus (GEO) dataset, and literature review. Significant upregulation of miR-106b-5p in cervical cancer is confirmed by meta-analysis with the data from TCGA, GEO, and literature. Moreover, the expression of miR-106b-5p is significantly correlated with the number of metastatic lymph nodes. Our bioinformatics analyses show that miR-106b could promote cervical cancer progression by modulating the expression of GSK3B, VEGFA, and PTK2 genes. Importantly, these three genes play a crucial role in PI3K-Akt signaling, focal adhesion, and cancer. Both the expression of miR-106b-5p and key genes are upregulated in cervical cancer. Several explanations could be implemented for this upregulation. However, the specific mechanism needs to be investigated further.
\end{abstract}

\section{Introduction}

At present, with an increase in morbidity and mortality, cancer has become the leading cause of death and a significant public health problem. There are over 500,000 novel cases and approximately 274,000 deaths estimated for cervical cancer (CC) each year all over the world ${ }^{1}$. In 2015, the number of new cases and deaths in China was $4,292,000$ and 2,814,000, respectively ${ }^{2}$. CC is the fourth most common cancer in women in the world, and incidence and mortality are still rising ${ }^{3}$. Although the relationship between persistent high-risk human papillomavirus (HPV) infection and CC has been confirmed ${ }^{4}$, the specific molecular cellular network mechanism is still unclear.

Genetic mutations lead to cancer by affecting gene expression and protein function in the cells. However, the dysregulation of microRNA (miRNA) expression is detected in a variety of tumors and is considered to be a significant contributor to the development of cancer in recent years ${ }^{5,6}$. miRNAs are small single-stranded non-

Correspondence: Wei Zhang (zw6676@163.com)

'Department of Obstetrics and Gynecology, Zhongnan Hospital of Wuhan University, Wuhan 430071 Hubei, People's Republic of China

Edited by: M.V. Niklison Chirou coding RNAs that specifically silence gene expression and alter cell or organism phenotypes. Previous studies have confirmed that miRNAs participate in proliferation, apoptosis, morphogenesis ${ }^{7}$, antiviral defense ${ }^{8}$, and tumorigenesis 9 .

Recently, growing evidence reveals that miR-106b-5p plays a critical role in various cancers. Huang and $\mathrm{Hu}^{10}$ showed that the upregulation of miR-106b can be observed in the endometrium and knockdown of miR106b suppresses proliferation and promotes apoptosis. Shi et al. ${ }^{11}$ reported that upregulation of miR-106b-5p exhibited a promoting role in hepatocellular carcinoma (HCC) cell properties and migration, whereas downregulation exhibited an opposite effect. Lu et al. ${ }^{12}$ and Xiang et al. ${ }^{13}$ submitted that overexpression of miR106b-5p could promote the proliferation and increase the number of metastatic colonies, whereas inhibition would induce cell cycle arrest, suppress cell proliferation, and promote cell apoptosis in renal cell carcinoma.

For CC, miR-106b, the pre-miRNA of miR-106b-5p, also has a pivotal role in occurrence and development. While constructing a miRNA-mRNA network for CC, $\mathrm{Ma}$

\section{(c) 2018 The Author(s).}

\footnotetext{
(c) (i) Open Access This article is licensed under a Creative Commons Attribution 4.0 International License, which permits use, sharing, adaptation, distribution and reproduction cc) in any medium or format, as long as you give appropriate credit to the original author(s) and the source, provide a link to the Creative Commons license, and indicate if changes were made. The images or other third party material in this article are included in the article's Creative Commons license, unless indicated otherwise in a credit line to the material. If material is not included in the article's Creative Commons license and your intended use is not permitted by statutory regulation or exceeds the permitted use, you will need to obtain permission directly from the copyright holder. To view a copy of this license, visit http://creativecommons.org/licenses/by/4.0/.
} 
Table 1 Correlations between expression of miR-106b-5p and clinical outcomes

\begin{tabular}{|c|c|c|c|c|}
\hline Item & Method & Cor. & $P$ value & FDR \\
\hline Number of lymph nodes & Kruskal-Wallis Test & 24.510 & $0.006^{\mathrm{a}}$ & 0.070 \\
\hline Tumor purity & Spearman Correlation & 0.107 & 0.078 & 0.430 \\
\hline Race & Kruskal-Wallis Test & 7.214 & 0.125 & 0.458 \\
\hline Pathology M stage & Wilcox Test & 0.025 & 0.193 & 0.530 \\
\hline Years to birth & Spearman Correlation & 0.063 & 0.300 & 0.553 \\
\hline Histological type & Kruskal-Wallis Test & 6.047 & 0.302 & 0.553 \\
\hline Ethnicity & Wilcox Test & -0.015 & 0.386 & 0.606 \\
\hline Radiation therapy & Wilcox Test & 0.011 & 0.545 & 0.750 \\
\hline Pathology N stage & Wilcox Test & -0.006 & 0.683 & 0.758 \\
\hline Pathology T stage & Kruskal-Wallis Test & 2.256 & 0.689 & 0.758 \\
\hline Overall survival & Cox Regression Test & 0.045 & 0.792 & 0.792 \\
\hline
\end{tabular}

${ }^{\text {a }}$ Significant difference

et al. ${ }^{14}$ found that miR-106b was one of the key nodes in the network. Overexpression of miR-106b promoted the migration of HeLa and SiHa cells significantly while inhibition displayed an opposite phenomenon ${ }^{15}$. However, few studies concern the mechanisms of miR-106b for $\mathrm{CC}$ at present. Cheng et al. ${ }^{15}$ found that DAB2 is identified as a direct target of miR-106b and it is inhibited by TGF- $\beta 1$ partly through miR-106b and is involved in TGF- $\beta 1$-induced CC cell migration. Piao et al. ${ }^{16}$ reported that miR-106 overexpression and DAB2 knockdown induced epithelial to mesenchymal transition (EMT) of CC cells cultured on substrate. As miR-106b plays an essential role in $\mathrm{CC}$, its molecular mechanisms need to be further studied.

The purpose of this study is to investigate the role of miR-106b-5p in CC by performing comprehensive research on its expression and identify its putative molecular targets and pathways based on The Cancer Genome Atlas (TCGA), Gene Expression Omnibus (GEO), and literature review.

\section{Results}

\section{Clinical significance of miR-106b-5p}

As the expression data of mature miR-106b-5p are absent in TCGA, a comparison of miR-106b between $\mathrm{CC}$ and healthy samples is provided. The expression level of miR-106b is higher in CC (Fig. 1) and significantly associated with the number of metastatic lymph nodes $($ Cor $=24.510, P=0.006)$. However, there is no significant correlation in tumor purity, race, pathological $\mathrm{M} / \mathrm{N} / \mathrm{T}$ stage, number of years of birth, histological type, race, radiotherapy, or overall survival (Table 1).

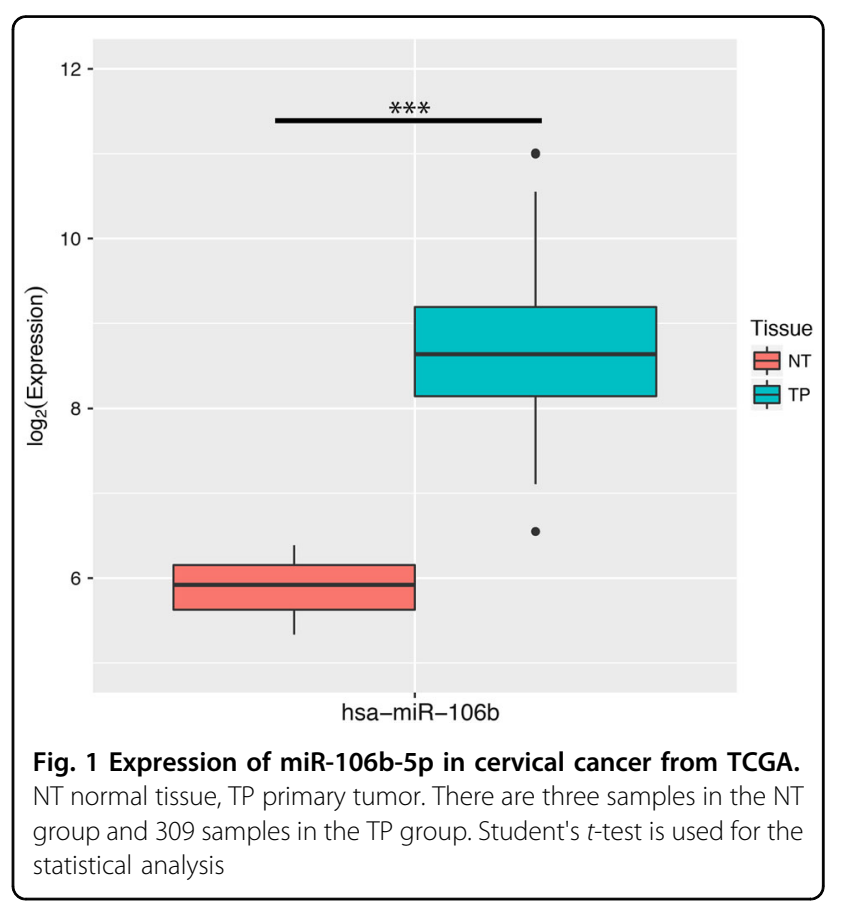

\section{Meta-analysis of miR-106b-5p expression Meta-analysis based on TCGA and GEO}

A total of 1286 microarrays were obtained from GEO. After careful screening, the three microarrays, GSE86100, GSE19611, and GSE30656, meet the criteria and are included in the analysis (Fig. 2a). The forest plot presents an overall standard mean difference (SMD) of 2.85 (95\% confidence interval (CI): $0.89-4.81$ ) with $P=0.0045$ and $I^{2}=88 \%$ (random effect used), suggesting that miR-106b$5 \mathrm{p}$ is upregulated in CC (Fig. 3a). 


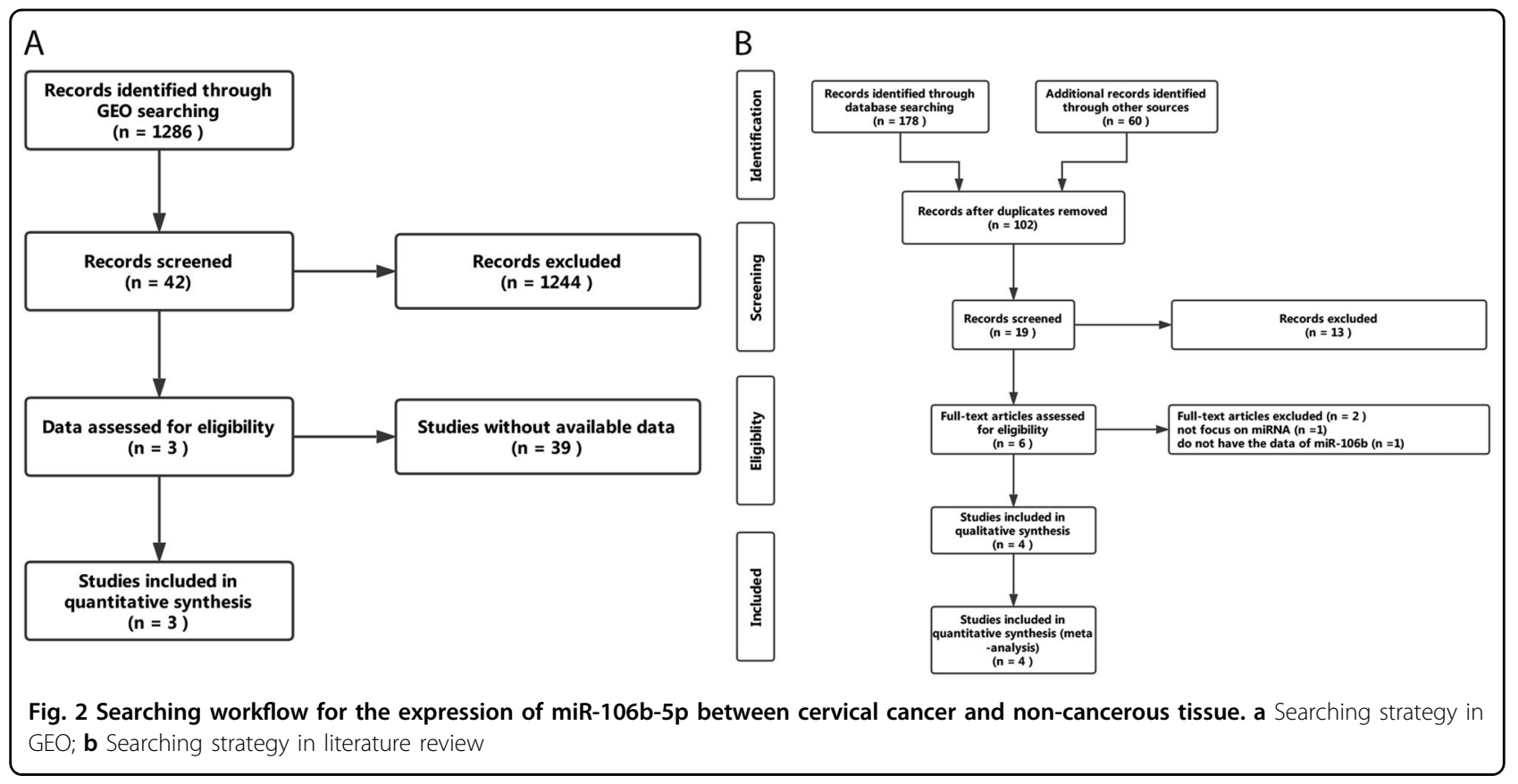

A funnel plot of miR-106b-5p expression (Fig. 3b) reveals that no significant publication bias is detected by Egger's test $(P=0.5187)$. Sensitivity analysis shows that similar results are obtained for the fixed effects models except for a lower difference (SMD: 2.53, 95\%CI: 1.89-3.18, $P<0.0001)$.

The influence analysis (Fig. 3c) shows that no study had an impact on the overall SMD estimation because the point estimate for any of the studies is not outside the combined analysis $\mathrm{CI}$ and there is no significant statistical change.

Except for a decrease in $I^{2}$ values, similar results are obtained in the subgroup analysis of cancer subtypes (Fig. 3d). The results show that the cancer subtype is not the only source of heterogeneity as the $I^{2}$ value is still relatively high, but miR-106b-5p continues to be highly expressed in CC tissues.

\section{Meta-analysis based on literature review}

The workflow for searching is presented in Fig. 2b. Finally, four studies ${ }^{14,15,17,18}$ that met the criteria were selected. Consistent with the result of the meta-analysis, a common pattern of upregulation for miR-106b-5p in CC was reported across the included studies (Table 2).

\section{Bioinformatics analyses of miR-106b-5p Screening of candidate genes}

By analyzing the data from Cervical squamous cell carcinoma and endocervical adenocarcinoma (CESC) with the criterion of $\log |\mathrm{FC}|>1$ and $\mathrm{FDR}<0.05,4857$ differentially expressed genes (DEGs) were selected, including 4619 upregulated genes and 238 downregulated genes. Meanwhile, predicting using 12 databases in miRWalk, 10,073 target genes that were overlapping in at least five databases were found (Fig. 4a). After merging DEGs and the predicted target genes, 1277 candidate genes were collected (Fig. 4b).

\section{Gene ontology enrichment analysis}

The DAVID database was used for Gene Ontology (GO) analysis of the 1277 genes (Fig. 5). Using the criterion of $P<0.001$, the results showed that while cellular component $(\mathrm{CeC})$, target genes are mainly involved in "cytoplasm", "cytosol", "receptor complex", "basolateral plasma membrane", "perinuclear region of cytoplasm", "ruffle membrane", "membrane", "lamellipodium", "cleavage furrow", "postsynaptic density", "membrane raft", "integral component of plasma membrane", and "cell cortex". In terms of biological process (BP), the target genes mainly participate in "protein phosphorylation", "positive regulation of transcription from RNA polymerase II promoter", "microtubule cytoskeleton organization", "epithelial to mesenchymal transition", "intracellular signal transduction", "cell migration", "protein autophosphorylation", and "positive regulation of protein binding". With regard to MF, these genes are mainly enriched in "protein binding", "protein serine/threonine kinase activity", "ATP binding", "kinase activity", "PDZ domain binding", and "transcription factor activity, sequence-specific DNA binding". 


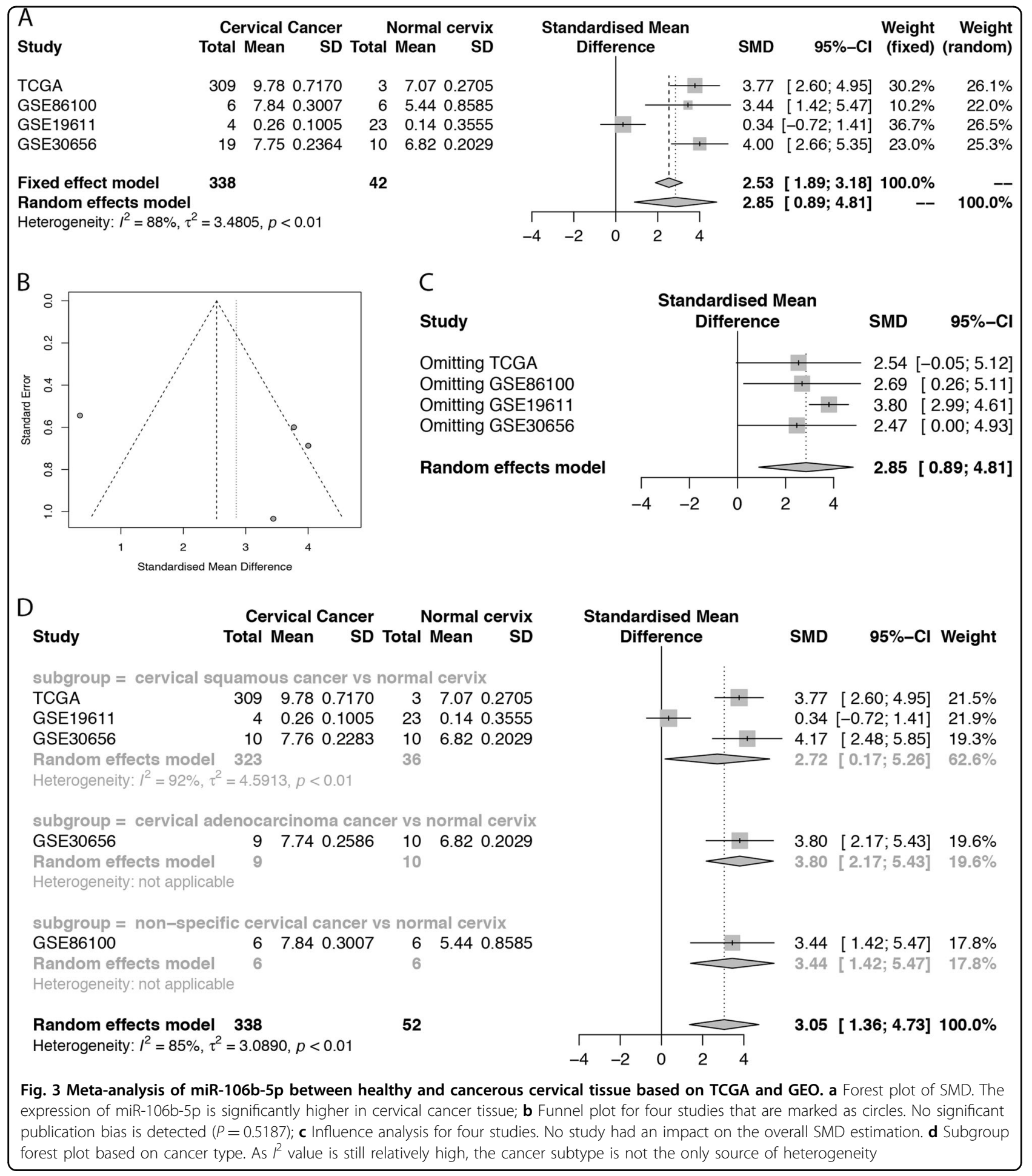

\section{Protein-protein interaction network analysis}

The original network contains 668 nodes and 1779 edges. After cleaning the isolated genes, the main network containing 579 nodes and 1716 edges was obtained (Fig. 6a). By extracting the nodes with degree and betweenness which are higher than average, a subnetwork that contains 110 nodes and 404 edges was gained (Fig. 6b; Table 3).

Twelve topological algorithms were applied and the top 20 genes of each method for the subnetwork of PPI were extracted. These selected genes that appeared at least twice are conserved as hub genes (Table 4). 
Table 2 Overview of the four studies selected in the literature review

\begin{tabular}{lllllll}
\hline Author & Year & Country & Cancer $(\boldsymbol{n})$ & Normal $(\boldsymbol{n})$ & Result & Detection methods \\
\hline Cheng et al. & 2016 & China & 19 & 19 & Upregulated & qRT-PCR \\
Gao et al. & 2016 & China & 30 & 26 & Upregulated & qRT-PCR \\
Ma et al. & 2012 & China & 8 & 8 & Upregulated & qRT-PCR \\
Liu et al. & 2016 & China & 10 & 10 & Upregulated & qRT-PCR \\
\hline
\end{tabular}

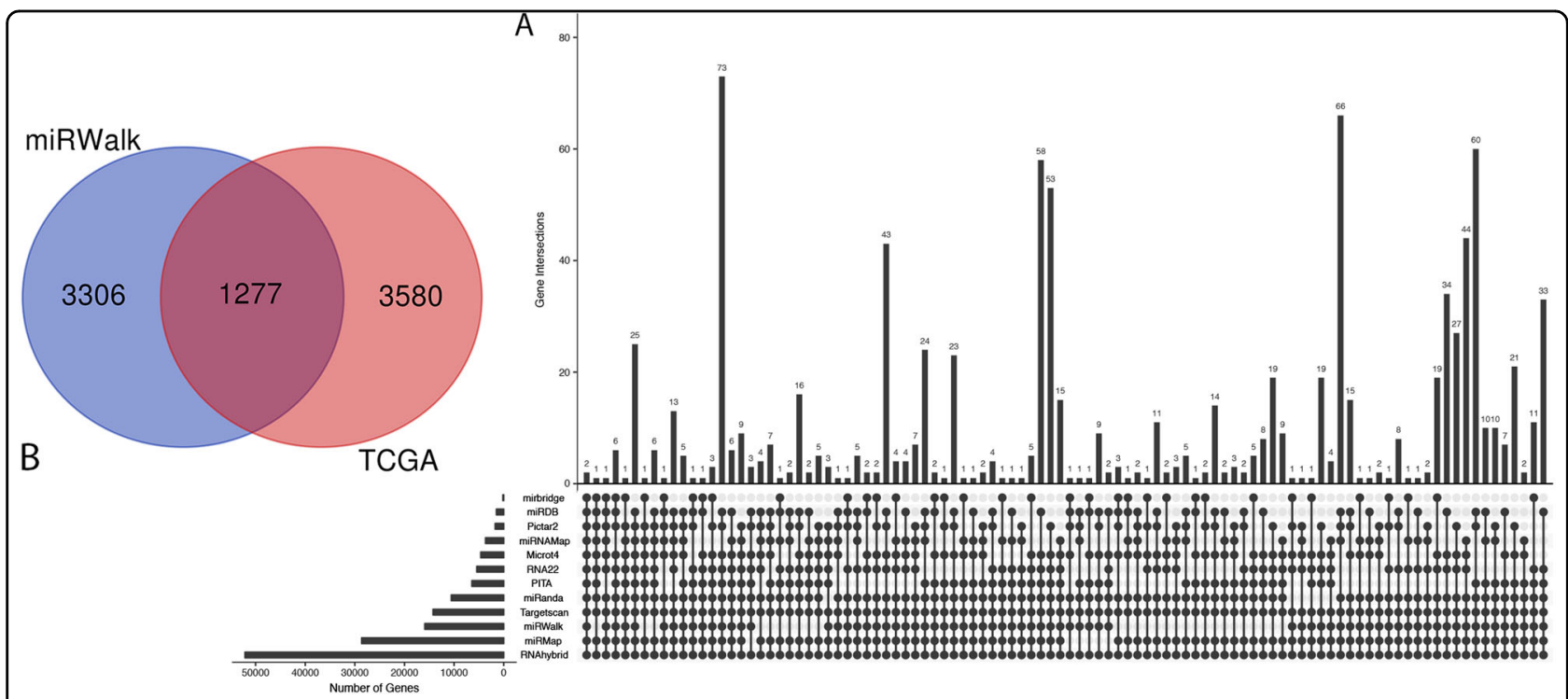

Fig. 4 Predication of miR-106b-5p target genes and candidate genes screening. a The number of overlapped genes across 12 databases; 10,073 target genes which overlapped at least five databases are obtained. $\mathbf{b}$ Venn plot for the integration between DEGs and predicted target genes of miR-106b-5p

\section{Pathway enrichment and crosstalk analysis}

Pathway enrichment analysis was also performed by the DAVID database. The results indicate that the pathways of "Signaling pathways regulating pluripotency of stem cells", "Neurotrophin signaling pathway", "Proteoglycans in cancer", and "Hippo signaling pathway" are significantly enriched.

For pathway crosstalk analysis, 45 out of 47 pathways that contain more than two genes met the crosstalk analysis criteria and were selected to construct the network (Fig. 6c). The thickness of the edges indicates measurements of the average value of $\mathrm{OC}$ and JC. By using MCODE, a major cluster with 33 nodes and 523 edges was identified from the initial network (Fig. 6d).

\section{Comprehensive gene-pathway analysis}

After mapping the hub genes into the subnetwork of pathways guided by KEGG, a potential gene-pathway network including 33 essential pathways and 16 hub genes were constructed (Fig. 6e). This network shows that "PIK3R3" and "PIK3CG" participate in most of the pathways. Furthermore, "pathways in cancer" and "focal adhesion" and "proteoglycans in cancer" rank as the top three pathways according to the genes that they involved.

To screen the main factors (including genes and pathways) in the gene-pathway network, those nodes with degree $>$ average were selected (Fig. 6f). It was found that 11 genes (PIK3R3, PIK3CG, MAPK8, GSK3B, ITGB1, CDKN1A, PTK2, VEGFA, PRKACB, RAP1B, and RAP1A) with five pathways (pathways in cancer, focal adhesion, proteoglycans in cancer, PI3K-Akt signaling pathway, and Ras signaling pathway) are involved and considered more likely to play a role as influential agents.

\section{Identification of key genes and pathways}

The expression of the main genes was investigated with meta-analysis through nine studies in Oncomine. The results show that all of the main genes are upregulated in CC, among which five genes (GSK3B, 

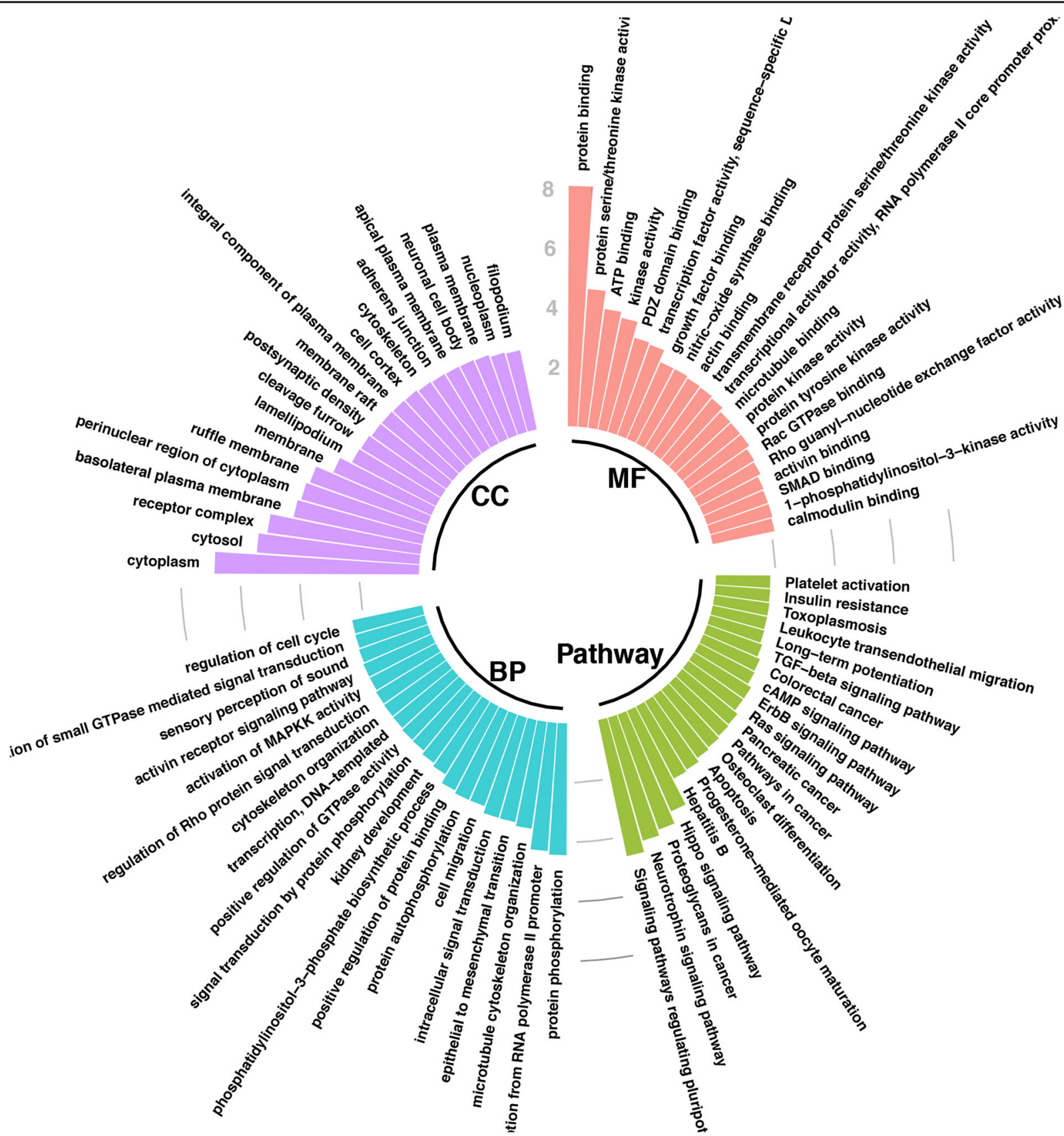

Fig. 5 The top 20 items of cellular component (CeC), biological process (BP) pathways, molecular function (MF), and pathways in Gene Ontology (GO) and pathway enrichment analysis for candidate target genes of miR-106b-5p in CC. Values are expressed as $-\log 10$ ( $P$-value)

VEGFA, PTK2, RAP1B, and PIK3CG) gain significant differences (Fig. 7a). However, the influence analysis indicates that RAP1B and PIK3CG obtain a discrepant result with the meta-analysis for the altering of significance (Table 5). Hence, only GSK3B, VEGFA, and PTK2 are considered to be key genes.

We merged the pathways that each essential gene participates in order to discover the most important pathways. Three pathways, PI3K-Akt signaling pathway, focal adhesion, and pathways in cancer, which all of the three key genes involved in, were identified (Fig. 7b).

\section{Location and characteristic of the binding site}

As presented in Fig. 7c, all of the binding sites for miR106b-5p are located in 3'UTR of GSK3B, VEGFA, and PTK2. Furthermore, when inspecting the sequence of binding sites, adenine (A) and uracil (U) are occupied in most of the sequence. 


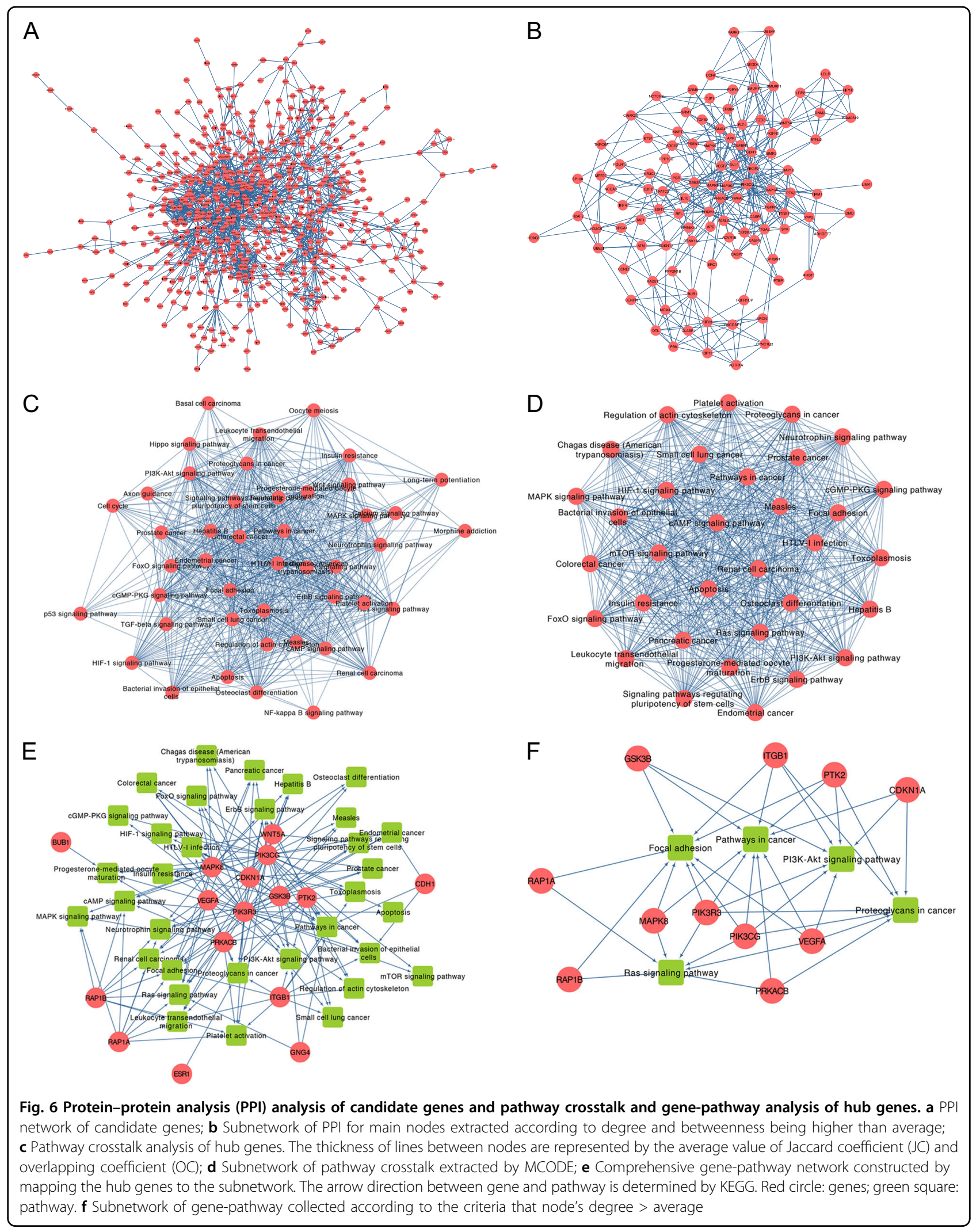


Table 3 Characteristics of main genes

\begin{tabular}{|c|c|c|}
\hline Gene & Degree & Betweenness \\
\hline Average value & 5.928 & 2064.408 \\
\hline PIK3CG & 46 & 41488.54899 \\
\hline PIK3R3 & 41 & 25199.73317 \\
\hline APP & 35 & 32767.23403 \\
\hline MAPK8 & 33 & 35071.18269 \\
\hline PRKACB & 29 & 27367.34287 \\
\hline PTK2 & 28 & 25355.409 \\
\hline ITGB1 & 28 & 17088.50374 \\
\hline SMURF1 & 27 & 7546.059618 \\
\hline SMURF2 & 27 & 6748.334913 \\
\hline BUB1 & 26 & 11066.28571 \\
\hline GNG4 & 26 & 10653.80228 \\
\hline H2AFV & 25 & 20305.78635 \\
\hline WNT5A & 25 & 13709.55435 \\
\hline VEGFA & 23 & 20760.79426 \\
\hline KIF23 & 21 & 10253.88184 \\
\hline RAD51 & 21 & 17892.93805 \\
\hline KIF11 & 21 & 5135.989346 \\
\hline CCNF & 20 & 5540.628454 \\
\hline RAP1A & 19 & 7521.72323 \\
\hline PPP2R1B & 19 & 14143.0877 \\
\hline BRCA1 & 19 & 6029.273042 \\
\hline NEDD4 & 19 & 3192.727805 \\
\hline CLASP1 & 19 & 7496.485807 \\
\hline SYK & 19 & 11386.15814 \\
\hline GSK3B & 19 & 18438.62749 \\
\hline DNM2 & 19 & 5911.698768 \\
\hline $\mathrm{ADCY} 2$ & 18 & 7560.286406 \\
\hline ATM & 18 & 9420.324388 \\
\hline CDKN1A & 18 & 18240.66944 \\
\hline RHOT1 & 18 & 16492.35297 \\
\hline HIP1R & 17 & 3517.329081 \\
\hline RAP1B & 17 & 5215.72323 \\
\hline MCM4 & 17 & 5808.281935 \\
\hline UBE4A & 17 & 2304 \\
\hline PARK2 & 17 & 2366.125584 \\
\hline $\mathrm{CDH} 1$ & 17 & 14376.3563 \\
\hline UBE2I & 17 & 10854.31473 \\
\hline SYNJ2 & 17 & 5273.61775 \\
\hline IL10 & 16 & 11158.43666 \\
\hline LRP2 & 16 & 3590.998058 \\
\hline KIAA0319 & 15 & 3475.143506 \\
\hline RACGAP1 & 15 & 5861.489126 \\
\hline ACTR1A & 15 & 3744.522591 \\
\hline PRDM10 & 15 & 14008.2127 \\
\hline FASLG & 15 & 5210.263877 \\
\hline BMP2 & 15 & 5337.049932 \\
\hline LDLR & 15 & 2191.70904 \\
\hline HDAC9 & 15 & 10403.97449 \\
\hline GRM5 & 14 & 3250.915796 \\
\hline CASP8 & 14 & 5874.766056 \\
\hline ESR1 & 14 & 11585.62988 \\
\hline NFATC2 & 14 & 4879.928683 \\
\hline PBK & 14 & 4821.131434 \\
\hline VAV2 & 14 & 8716.774939 \\
\hline GRM1 & 14 & 3250.915796 \\
\hline
\end{tabular}

Table 3 continued

\begin{tabular}{|c|c|c|}
\hline Gene & Degree & Betweenness \\
\hline FGFR1OP & 13 & 6727.611655 \\
\hline DTL & 13 & 2540.356126 \\
\hline TGFBR1 & 13 & 4022.943631 \\
\hline CAMK2D & 13 & 5055.739472 \\
\hline CASP9 & 12 & 4919.739038 \\
\hline P2RY6 & 12 & 2304 \\
\hline YWHAZ & 11 & 4040.292887 \\
\hline MAP3K7 & 11 & 6201.810194 \\
\hline FLT1 & 11 & 6634.16899 \\
\hline PPP1CB & 11 & 2005.113067 \\
\hline NCOA3 & 11 & 7457.814126 \\
\hline FGFR2 & 11 & 3030.464668 \\
\hline ITGA2 & 10 & 3529.023629 \\
\hline NOTCH2 & 10 & 10947.19567 \\
\hline CENPN & 10 & 1873.156984 \\
\hline FGFR1 & 10 & 8007.32537 \\
\hline IRF4 & 10 & 5202.998605 \\
\hline PTBP1 & 10 & 6730.910039 \\
\hline MEF2C & 10 & 4165.497288 \\
\hline ARHGEF7 & 9 & 3147.468396 \\
\hline TIAM1 & 9 & 4058.542014 \\
\hline ERBB4 & 9 & 4519.484052 \\
\hline ARCN1 & 9 & 2088.364196 \\
\hline CASP7 & 9 & 5818.243304 \\
\hline PGR & 9 & 4778.496029 \\
\hline SPTBN1 & 9 & 10172.1474 \\
\hline DMD & 9 & 2456.15705 \\
\hline FZD3 & 9 & 2260.879218 \\
\hline MAPK9 & 9 & 2693.578423 \\
\hline DYNC1LI2 & 9 & 2495.446083 \\
\hline NR3C1 & 9 & 4868.398944 \\
\hline DVL3 & 9 & 3925.165711 \\
\hline HDAC8 & 9 & 2385.834093 \\
\hline APC & 9 & 4483.983598 \\
\hline TNRC6A & 8 & 2766.776691 \\
\hline RPS6KA1 & 8 & 2691.499073 \\
\hline TJP1 & 8 & 4534.026739 \\
\hline ACVR1B & 8 & 1933.546424 \\
\hline TGFB2 & 8 & 2116.806735 \\
\hline SP100 & 8 & 2757.903141 \\
\hline POLR1E & 7 & 6748.802196 \\
\hline ETS1 & 7 & 4087.280316 \\
\hline CSNK1A1 & 7 & 2157.303406 \\
\hline PSEN1 & 7 & 3233.020264 \\
\hline TAF1 & 7 & 4214.786588 \\
\hline ERC1 & 7 & 3537.432599 \\
\hline CCNE2 & 7 & 1750.370853 \\
\hline REL & 7 & 2794.403409 \\
\hline MAPT & 7 & 2044.275299 \\
\hline LIMK1 & 7 & 1750.999958 \\
\hline CSF2RA & 7 & 1782.306466 \\
\hline E2F2 & 7 & 4585.295895 \\
\hline
\end{tabular}


Table 4 Hub genes identified from top 20 of 12 topological algorithms

\begin{tabular}{|c|c|c|}
\hline Rank & Gene & Counts \\
\hline 1 & APP & 9 \\
\hline 2 & MAPK8 & 9 \\
\hline 3 & PIK3CG & 9 \\
\hline 4 & PIK3R3 & 9 \\
\hline 5 & VEGFA & 9 \\
\hline 6 & ITGB1 & 8 \\
\hline 7 & PRKACB & 8 \\
\hline 8 & PTK2 & 8 \\
\hline 9 & GNG4 & 6 \\
\hline 10 & GSK3B & 6 \\
\hline 11 & PRDM10 & 6 \\
\hline 12 & WNT5A & 6 \\
\hline 13 & CDKN1A & 5 \\
\hline 14 & RAD51 & 5 \\
\hline 15 & SMURF1 & 5 \\
\hline 16 & SMURF2 & 5 \\
\hline 17 & CCNF & 4 \\
\hline 18 & $\mathrm{CDH} 1$ & 4 \\
\hline 19 & ESR1 & 4 \\
\hline 20 & H2AFV & 4 \\
\hline 21 & NEDD4 & 4 \\
\hline 22 & TRIM36 & 4 \\
\hline 23 & BUB1 & 3 \\
\hline 24 & EHHADH & 3 \\
\hline 25 & FBXL5 & 3 \\
\hline 26 & RAP1A & 3 \\
\hline 27 & RAP1B & 3 \\
\hline 28 & RHOT1 & 3 \\
\hline 29 & AGFG1 & 2 \\
\hline 30 & ASB13 & 2 \\
\hline 31 & FASLG & 2 \\
\hline 32 & FLT1 & 2 \\
\hline 33 & KBTBD8 & 2 \\
\hline 34 & KIAA0319 & 2 \\
\hline 35 & KIF11 & 2 \\
\hline 36 & $\mathrm{KIF} 23$ & 2 \\
\hline 37 & KLHL20 & 2 \\
\hline 38 & KLHL5 & 2 \\
\hline
\end{tabular}

Table 4 continued

\begin{tabular}{lll}
\hline Rank & Gene & Counts \\
\hline 39 & LDLR & 2 \\
40 & LRP2 & 2 \\
41 & MKRN1 & 2 \\
42 & PACSIN1 & 2 \\
43 & PARK2 & 2 \\
44 & PCYT1B & 2 \\
45 & PGR & 2 \\
46 & RLIM & 2 \\
47 & RNF213 & 2 \\
48 & SPSB4 & 2 \\
49 & UBE4A & 2 \\
\hline
\end{tabular}

\section{Discussion}

This study confirms that the expression of miR-106b$5 p$ is significantly upregulated in $\mathrm{CC}$ and its expression is highly correlated with the number of metastatic lymph nodes. By using bioinformatics analyses, miR-106b-5p is found to be a key issue in the progression of $\mathrm{CC}$ by interacting with three key genes and pathways.

Our results show that miR-106b-5p promotes the progression of CC by targeting GSK3B, VEGFA, and PTK2. GSK3B is dysregulated in a variety of tumor tissues including $\mathrm{CC}^{19-24}$, and it participates in the development of CC caused by HPV16 infection by regulating Wnt signaling $/ \beta$-catenin pathway ${ }^{23,24}$. In addition, GSK3B exerts antiproliferative effects by promoting APCdependent phosphorylation and thus promotes proteasemediated degradation of $\beta$-catenin which is a transcription factor that positively regulates Myc and cyclin D1 expression $^{21}$. When referring to VEGFA, its overexpression in CC can enhance the growth and invasion of tumor cells ${ }^{25}$. More importantly, VEGFA can promote the proliferation and migration of $\mathrm{CC}$ cells by activating the PI3K/Akt/mTOR pathway ${ }^{26}$. In addition, the expression and stability of VEGFA have a close relationship with cellular hypoxia ${ }^{27}$ and serum VEGFA level can be used as a biomarker for prognosis evaluation ${ }^{28}$. In terms of PTK2, it is a cytoplasmic protein tyrosine kinase and has an expression in various solid tumors such as ovarian cancer, gastric cancer, and bladder cancer ${ }^{29}$. It is found that PTK2 is associated with the sensitivity of colon cancer cells to DNA damage therapy ${ }^{30}$. However, there are few reports of PTK2 in CC.

The results of pathway analyses reveal that all of the three key genes are involved in the pathway of PI3K-Akt signaling pathway, focal adhesion, and Pathways in 


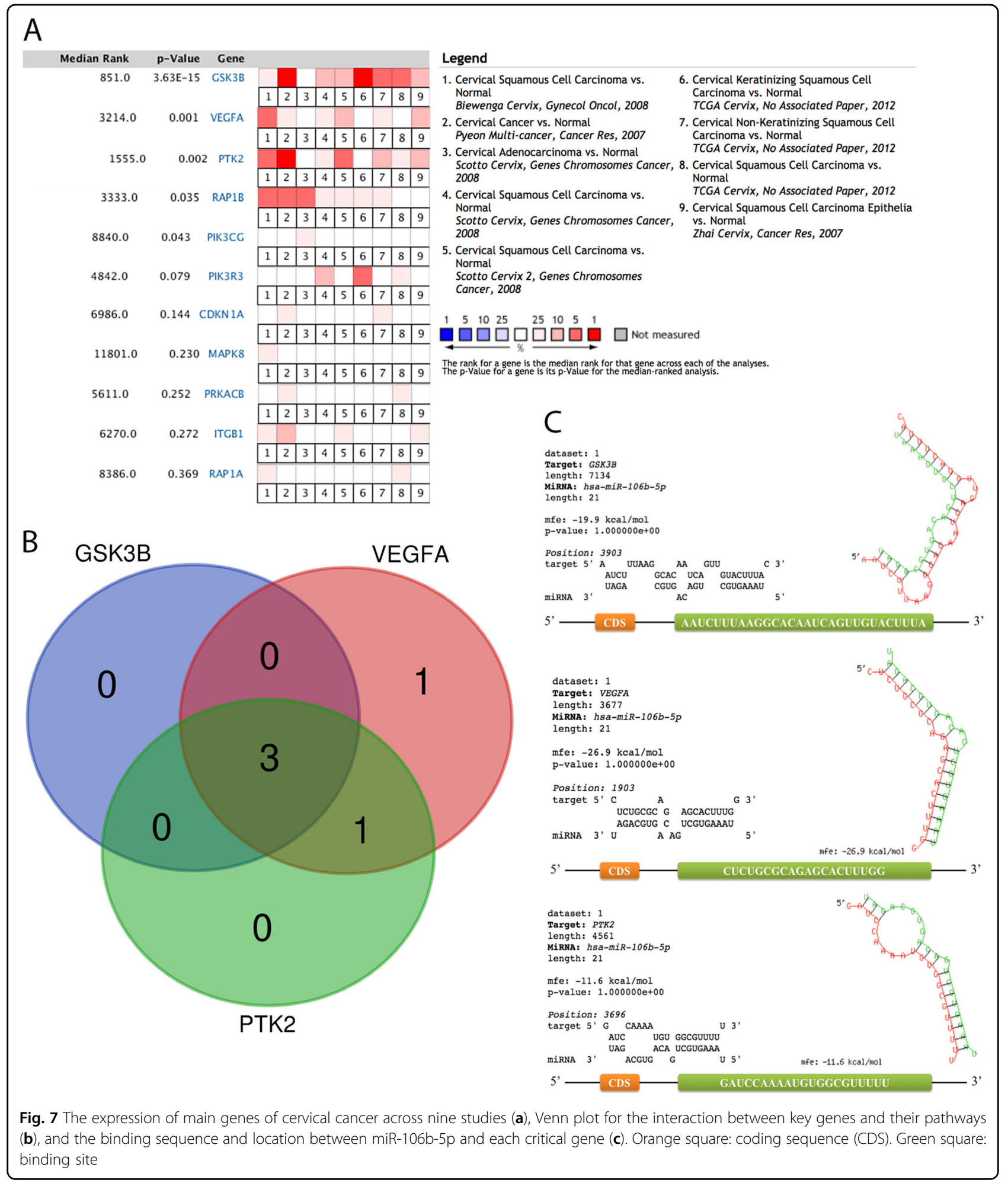

cancer. With regard to PI3K-Akt signaling pathway, its imbalance in expression is associated with a variety of tumors, including cervical, endometrial, and non-small cell lung cancers ${ }^{31-33}$. It is activated during the G1/S transition of the cell cycle and regulates several key cell cycle regulators ${ }^{34}$. Tumors associated with HPV infection are closely related to the PI3K/Akt pathway. Activation of this pathway contributes to genetic instability, dysregulation, apoptosis resistance, and altered metabolic properties ultimately leading to the malignant transformation 
of infected cells. Concerning focal adhesion, this pathway is involved in the invasion and metastasis of many kinds of tumors and is related to the medicine resistance of certain tumors $^{35,36}$. The decreased expression of focal adhesion kinase (FAK), a key gene involved in the pathway, can inhibit the invasion and migration of $\mathrm{CC}$ cells $^{37}$. Another study reports that targeted FAK therapy makes pancreatic cancer cells more sensitive to immunotherapy ${ }^{38}$.

It has been recognized that miRNA negatively regulates gene expression by guiding the RNA-induced silencing complex (RISC) to silence its target mRNA through degradation or translation repression ${ }^{39}$. However, it is interesting to observe that both the expression of miR106b-5p and key genes are upregulated in CC with the binding sites located in $3^{\prime} U T R$ of mRNAs. According to previous studies ${ }^{40-44}$, the mechanisms of miRNAmediated gene upregulation include (1) the presence of cellular state (G0 or G0-like state) and/or specific factors (AGO1, AGO2, GW182, and FXR1); (2) miRNA directly binds to $5^{\prime}$ UTR of RNA and increases its association with $40 \mathrm{~S}$ and polysome formation or enhances their translation by alleviating their TOP-mediated translational repression during amino acid starvation, respectively; (3) miRNA prevents tristetraprolin (TTP) binding to the AU-rich element (ARE) sites of mRNA and inhibits its degradation by ARE-mediated mRNA decay (AMD) pathway; (4) derepression from miRNA-mediated downregulation in response to cell stresses by HuR (an RNA binding protein that interacts with ARE in 3'UTR of the mRNA). As none of the binding sites are located in $5^{\prime} \mathrm{UTR}$ in our results, and cells are considered active in cancer tissue, the mechanisms of upregulation for cellular state or binding site in $5^{\prime}$ UTR may be less possible.

AREs are found in the $3^{\prime}$ UTR of mRNAs that code for proto-oncogenes, nuclear transcription factors, and cytokines ${ }^{45}$. They can be classified into three types: (1) having dispersed AUUUA motifs within or near U-rich regions; (2) having overlapping AUUUA motifs within or near U-rich regions; (3) a much less well-defined class having a U-rich region but no AUUUA repeats ${ }^{46}$. Our results reveal that $A$ and $U$ are occupied in most of the binding sequence. To our knowledge, it has been verified that TTP has interactions with $\mathrm{GSK} \mathrm{B}^{47}$ and VEGFA $^{48}$ whereas there are no reports on PTK2. It is possible that miR-106b-5p prevents TTP from binding to the mRNAs in $3^{\prime} U T R$ and therefore regulate their expression, but the specific mechanism needs to be further investigated. In addition, it has been confirmed that $\mathrm{HuR}$ can bind to VEGF ${ }^{49}$ and reverse the repression effect by miR-200b ${ }^{50}$. This also could be a contributing factor to the upregulation of VEGFA and be adopted for the other key genes.

However, several limitations exist in the present study: (1) Significant heterogeneity can be observed in the meta-analysis. This high heterogeneity may result from population, race, stage, and the type of CC (squamous or adenocarcinoma). Hence, more data from large-scale clinical trials are needed to evaluate the source of the heterogeneity. (2) Parts of genes are removed due to the selection criteria. However, it is possible that these genes might also impact the progression of $\mathrm{CC}$ and they also need to be analyzed. (3) As this study is an in silico research, a further experiment is needed for validation. (4) Upregulations of both miR-106-5p and key genes are identified. Despite the fact that several possibilities are analyzed, the specific mechanisms still need to be studied and verified further.

\section{Conclusions}

In summary, significant upregulation of miR-106b-5p in $\mathrm{CC}$ is confirmed by meta-analysis with the data from TCGA and GEO, and the expression of miR-106b-5p is significantly correlated with the number of metastatic lymph nodes. Furthermore, miR-106b-5p promotes the progression of $\mathrm{CC}$ by targeting three key genes (GSK3B, VEGFA, PTK2) through three crucial pathways (PI3K-Akt signaling pathway, focal adhesion, and pathways in cancer). miR-106b-5p might upregulate the key genes by preventing TTP from binding to the mRNAs in 3'UTR with/without the effect of derepression of HuR. However, the specific mechanism needs to be further investigated.

\section{Materials and methods}

The workflow of this study is presented in Fig. 8 and this study is performed according to guidelines of MIAME $^{51}$ and Meta-Analysis of Gene Expression Microarray Datasets ${ }^{52}$. Firstly, the clinical significance of miR-106b-5p in CC is assessed according to the CESC data from TCGA. Secondly, the expression data of miR106b-5p from TCGA, GEO, and literature are synthesized by meta-analysis. Thirdly, DEGs from TCGA are screened and 12 databases are used to predict target genes of miR-106b-5p. The overlap genes between DEGs from TCGA and predicted target genes are explored by bioinformatic analyses.

\section{Correlations between the expression of miR-106b-5p and clinical outcomes}

To identify the clinical significance of miR-106b-5p in $\mathrm{CC}$, we adopt LinkedOmics ${ }^{53}$ which is constructed based on TCGA with 309 cancer samples and three normal samples as controls in CESC dataset to explore the correlations between the expression of miR-106b-5p and the clinical outcomes, including the number of metastatic lymph nodes, tumor purity, race, pathology $\mathrm{M} / \mathrm{N} / \mathrm{T}$ stage, years to birth, histological type, ethnicity, radiation therapy, and overall survival. 


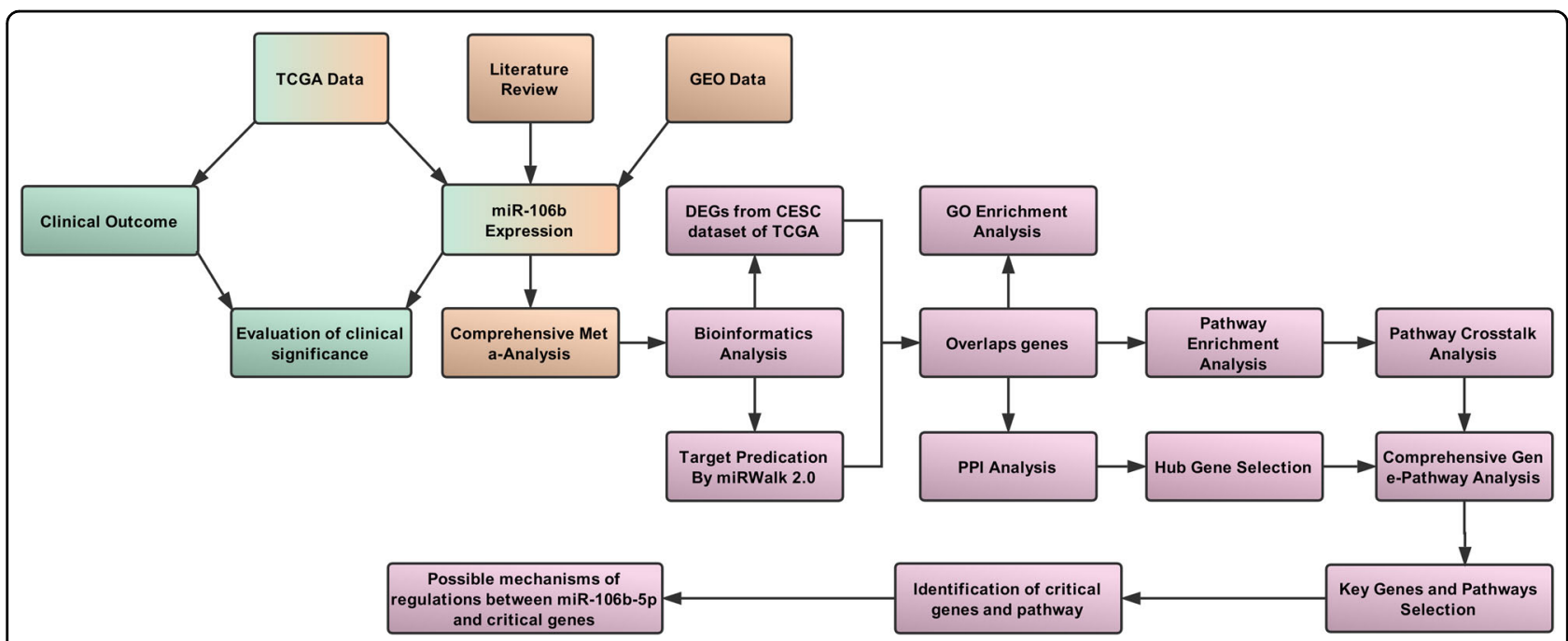

Fig. 8 Work flow of the clinical significance evaluation and comprehensive analysis for miR-106b-5p in cervical cancer. The modules of clinical value evaluation (green), meta-analysis (brown), and bioinformatics analyses (pink) are included

\section{Meta-analysis of miR-106b-5p expression based on TCGA/ GEO and literature}

A systematic searching in GEO for the miR-106b-5p expression between $\mathrm{CC}$ and healthy tissue was performed on 30 March 2018 with the terms and eligibility criteria that are presented in Tables 5 and 6. For adjusting the data to the normal distribution to reduce variation, Log2 scale transformation was applied to all of the expression data. The meta-analysis was conducted by $\mathrm{R}$ Version $3.4 .1^{54}$ and meta package ${ }^{55}$. We pooled SMD using the Mantel-Haenszel formula (fixed-effect model) or the DerSimonian-Laird formula (random effects model) and assessed the heterogeneity using $I^{2}$ statistics. Random-effect models $\left(P<0.05\right.$ or $\left.I^{2}>50 \%\right)$ are used when the heterogeneity is considered significant. Otherwise a fixed-effects model would be used.

Funnel plot with Egger's test ${ }^{56}$ was utilized to evaluate the publication bias. $P<0.1$ was considered to be significant asymmetry for the funnel plot.

To detect the robustness of the pool results, sensitivity analysis was performed by alternating analysis model. In addition, to further evaluate the impact of individual studies on the overall effect estimates, influence analysis was performed and the combined estimates were recalculated by omitting one study at a time.

Furthermore, a full-scale search of miR-106b-5p expression in eight electronic databases (PubMed, Chinese VIP, CNKI, Wanfang Database, Embase, Web of Science, Science Direct, and Wiley Online Library) accompanied with manual searching by screening the references cited in the acquired articles was conducted on 30 March 2018. The searching terms and eligible criteria are also shown in Tables 5 and 6 . The data of the
Table 5 Influence analysis of 5 key genes

\begin{tabular}{llllll}
\hline Omitting study & $\mathbf{P}_{\text {GSK3B }}$ & $\mathbf{P}_{\text {VEGFA }}$ & $\mathbf{P}_{\mathrm{PTK} 2}$ & $\mathbf{P}_{\mathrm{RAP1B}}$ & $\mathbf{P}_{\mathrm{PIK3CG}}$ \\
\hline Omitting Study 1 & $2.16^{*} 10^{-6}$ & 0.023 & $9.22^{*} 10^{-4}$ & 0.067 & 0.165 \\
Omitting Study 2 & $1.88^{*} 10^{-4}$ & 0.023 & $9.22^{*} 10^{-4}$ & 0.067 & 0.489 \\
Omitting Study 3 & $2.16^{*} 10^{-6}$ & $7.55^{*} 10^{-4}$ & $9.51^{*} 10^{-4}$ & 0.067 & 0.367 \\
Omitting Study 4 & $2.16^{*} 10^{-6}$ & 0.022 & $9.51^{*} 10^{-4}$ & 0.023 & 0.165 \\
Omitting Study 5 & $1.88^{*} 10^{-4}$ & 0.023 & $9.22^{*} 10^{-4}$ & 0.067 & 0.367 \\
Omitting Study 6 & $1.88^{*} 10^{-4}$ & $7.55^{*} 10^{-4}$ & $9.51^{*} 10^{-4}$ & 0.023 & 0.165 \\
Omitting Study 7 & $1.88^{*} 10^{-4}$ & $7.55^{*} 10^{-4}$ & $2.97^{*} 10^{-5}$ & 0.055 & 0.367 \\
Omitting Study 8 & $1.90^{*} 10^{-4}$ & $7.55^{*} 10^{-4}$ & $9.51^{*} 10^{-4}$ & 0.023 & 0.165 \\
Omitting Study 9 & $2.16^{*} 10^{-6}$ & 0.023 & $9.22^{*} 10^{-4}$ & 0.023 & 0.367 \\
\hline
\end{tabular}

Study 1: Cervical Squamous Cell Carcinoma vs. Normal .Biewenga Cervix, Gynecol Oncol, 2008

Study 2: Cervical Cancer vs. Normal. Pyeon Multi-cancer, Cancer Res, 2007

Study 3: Cervical Adenocarcinoma vs. Normal. Scotto Cervix, Genes Chromosomes Cancer, 2008

Study 4: Cervical Squamous Cell Carcinoma vs. Normal. Scotto Cervix, Genes Chromosomes Cancer, 2008

Study 5: Cervical Squamous Cell Carcinoma vs. Normal. Scotto Cervix 2, Genes Chromosomes Cancer, 2008

Study 6: Cervical Keratinizing Squamous Cell Carcinoma vs. Normal. TCGA Cervix, No Associated Paper, 2012

Study 7: Cervical Non-Keratinizing Squamous Cell Carcinoma vs. Normal. TCGA Cervix, No Associated Paper, 2012

Study 8: Cervical Squamous Cell Carcinoma vs. Normal. TCGA Cervix, No Associated Paper, 2012

Study 9: Cervical Squamous Cell Carcinoma Epithelia vs. Normal. Zhai Cervix, Cancer Res, 2007.

$\mathrm{P}_{\text {Gene: }}$ The $\mathrm{p}$-Value for a gene and is the $\mathrm{p}$-Value for the median-ranked analysis

publications will be extracted and analyzed, including the author, year of publication, country, number of cancer and control samples, regulations, and testing methods. 


\section{Screening of candidate genes for miR-106b-5p}

The CESC data were analyzed by the limma package ${ }^{57}$ to identify DEGs. We determined the significance of the

\section{Table 6 Searching terms used in GEO and literature review}

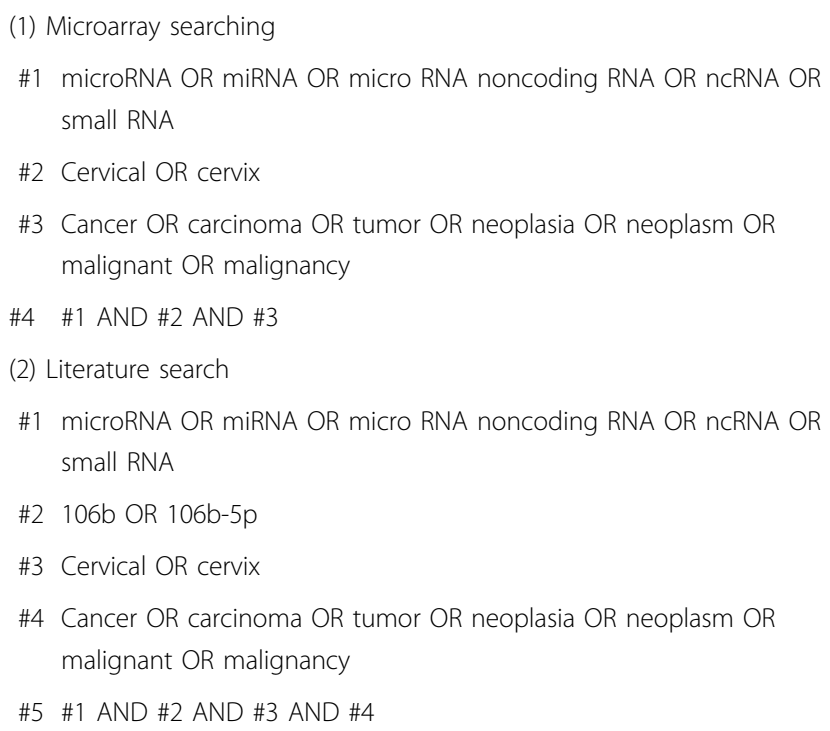

difference in gene expression as Log2 |fold change (FC) | $>1$ and False Discovery Rate (FDR) $<0.05$. Furthermore, the miR-106b-5p targeted genes are predicted by 12 databases (Microt4, miRWalk, mir-bridge, miRanda, miRDB, miRMap, Pictar2, PITA, miRNAMap, RNAhybrid, RNA22, and Targetscan) in miRWALK version $2.0^{58}$. To increase prediction accuracy, the genes that are overlapping in at least five databases were selected. Finally, the overlap genes between DEGs and predicted genes were analyzed by upset $R^{59}$ and Venn Plot.

\section{GO and pathway enrichment analysis}

GO and pathway enrichment analyses were performed by DAVID ${ }^{60}$ on the overlapping genes. The significantly enriched biological items for $\mathrm{CeC}, \mathrm{BP}$, and molecular functions (MF) were identified as $P<0.01$.

\section{Protein-protein interaction network analysis}

The STRING database ${ }^{61}$ was used to construct an interaction network between the overlapped genes. To obtain precise results, those nodes in the network would be removed: (a) interaction score $<0.7$; (b) not connected to the major network; (3) the value of degree and betweenness is less than average.

CytoHubba ${ }^{62}$ was used to explore the important nodes by 12 topological algorithms, including Degree, Edge

Table 7 Eligibility criteria of inclusion and exclusion for GEO and literature review

\section{Eligibility criteria for GEO}

(1) Inclusion criteria

(a) CC tissues are included in each dataset with each group containing more than two samples, regardless of the inclusion of adjacent noncancerous tissues (or healthy tissues)

(b) The dataset sample organism is Homo sapiens

(c) The expression data of miR-106b (hsa-miR-106b or hsa-miR-106b-5p) from the experimental and control groups could be provided or calculated

(2) Exclusion criteria
(a) Datasets without information on miR-106b or miR-106b-5p
(b) Datasets without complete data for analysis
(c) Samples based on cell lines
(d) Not all the subjects of the included studies are human
(e) miR-106b-5p is determined in the CC patients without a comparison
(f) The original paper which the dataset related to cannot be achieved

2. Eligibility criteria for literature review

(1) Inclusion criteria

(a) Studies examining the expression of miR-106b in CC

(b) Normal cervical tissues paired/unpaired used as healthy control group

(2) Exclusion criteria

(a) Reviews, experimental studies, single case reports, meta-analyses, and conference abstracts

(b) Absence of healthy control groups 
Percolated Component (EPC), Maximum Neighborhood Component (MNC), Density of Maximum Neighborhood Component (DMNC), Maximal Clique Centrality (MCC), and centralities based on shortest paths, such as Bottleneck (BN), EcCentricity, Closeness, Radiality, Betweenness, ClusteringCoefficient, and Stress. The top 20 genes in each topological algorithm were extracted and the duplication of each gene was also calculated. The genes with less than 2 in repetitiveness are excluded for ensuring the genes are closely linked to CC and the rest are considered as hub genes.

\section{Pathway enrichment and crosstalk analysis}

For pathway enrichment, the predicted targets were mapped using the Kyoto Gene and Genome Encyclopedia (KEGG) database by online analysis of DAVID. Significant pathways were considered as $P<0.05$.

The obtained pathways were recruited for further crosstalk analysis to explore their interactions. The method is based on the assumption that if two pathways share a proportion of genes, they are considered crosstalk $^{63}$. As described in the previous study ${ }^{64}$, in order to better measure the overlap between any two pathways, JC (Jaccard coefficient $)=|(A \cap B) /(A \cup B)|$ and $O C$ (overlapping coefficient $)=(|A \cap B|) /(\min (|A|,|B|))$ were adopted, where $A$ and $B$ are the genes contained in the two pathways. As pathways with too few genes may have insufficient biological information, we excluded pathways containing fewer than three genes. Likewise, pairs of pathways with less than two overlapping genes were also removed. After obtaining the network of pathway crosstalk, the plug-in app Molecular Complex Detection (MCODE) in Cytoscape was used to screen the hub subnetwork which had the score $>4$.

\section{Comprehensive gene-pathway analysis}

The hub genes are mapped into the subnetwork of crosstalk to further explore the mechanism by KEGG. To screen the main genes and pathways, the nodes with degree $>$ average are collected for constituting a subnetwork.

\section{Identification of key genes and pathways}

To further identify the key genes, we evaluated the expression of main genes between $\mathrm{CC}$ and healthy samples with meta-analysis in Oncomine ${ }^{65} . P<0.05$ is considered as a significant difference. Moreover, influence analysis was also conducted to access the pool estimates. The pathways that all of the key genes participate in were determined as crucial pathways.

\section{Possible mechanisms of regulations between miR-106b-5p and key genes}

The regulation between miR-106b-5p and the key genes is recognized by their expression. To explore the possible mechanisms of the regulation, we collected the sequence of miR-106b-5p and the three key genes from miRbase ${ }^{66}$ and NCBI-nucleotide to predict their binding site by RNAhybrid $^{67}$ with the criteria that mRNA has perfect nucleotide pairing between the second and eighth positions of the $5^{\prime}$ end of miRNA sequences. Furthermore, the character of the binding sequence was also investigated.

\section{Conflict of interest}

The authors declare that they have no conflict of interest.

\section{Publisher's note}

Springer Nature remains neutral with regard to jurisdictional claims in published maps and institutional affiliations.

The online version of this article (https://doi.org/10.1038/s41420-018-0096-8) contains supplementary material, which is available to authorized users.

Received: 13 June 2018 Revised: 29 July 2018 Accepted: 6 August 2018 Published online: 20 September 2018

\section{References}

1. Torre, L. A. et al. Global Cancer Statistics, 2012. CA Cancer J. Clin. 65, 87-108 (2015).

2. Chen, W. et al. Cancer Statistics in China. CA Cancer J. Clin. 66, 115-132 (2016).

3. Siegel, R. L., Miller, K. D. \& Jemal, A. Cancer statistics, 2018. CA Cancer J. Clin. 68, 7-30 (2018).

4. Petry, K. U. HPV and cervical cancer. Scand. J. Clin. Lab. Invest. 74, 59-62 (2014).

5. Wang, T., Xu, H., Qi, M., Yan, S. \& Tian, X. miRNA dysregulation and the risk of metastasis and invasion in papillary thyroid cancer: a systematic review and meta-analysis. Oncotarget 9, 5473-5479 (2018)

6. Santos, J. M. O., Gil da Costa, R. M. \& Medeiros, R. Dysregulation of cellular microRNAs by human oncogenic viruses - Implications for tumorigenesis. Biochim. Biophys. Acta 1861, 95-105 (2018).

7. Kim, V. N. Small RNAs: classification, biogenesis, and function. Mol. Cells 19, 1-15 (2005).

8. Lecellier, C.-H. A cellular microRNA mediates antiviral defense in human cells. Science 308, 557-560 (2005).

9. O'Donnell, K. A., Wentzel, E. A., Zeller, K. I., Dang, C. V. \& Mendell, J. T. c-Mycregulated microRNAs modulate E2F1 expression. Nature 435, 839-843 (2005).

10. Huang, C. \& Hu, G. Shikonin suppresses proliferation and induces apoptosis in endometrioid endometrial cancer cells via modulating miR-106b/PTEN/AKT/ mTOR signaling pathway. Biosci. Rep. 0, BSR20171546 (2018).

11. Shi, D. M., Bian, X. Y., Qin, C. D. \& Wu, W. Z. miR-106b-5p promotes stem celllike properties of hepatocellular carcinoma cells by targeting PTEN via PI3KJAkt pathway. Onco. Targets Ther. 11, 571-585 (2018).

12. Lu, J. et al. miR-106b-5p promotes renal cell carcinoma aggressiveness and stem-cell-like phenotype by activating Wnt/B-catenin signalling. Oncotarget $\mathbf{8}$, 21461-21471 (2017).

13. Xiang, W. et al. miR-106b-5p targets tumor suppressor gene SETD2 to inactive its function in clear cell renal cell carcinoma. Oncotarget 6, 4066-4079 (2015).

14. Ma, D., Zhang, Y. Y., Guo, Y. L., Li, Z. J. \& Geng, L. Profiling of microRNA-mRNA reveals roles of microRNAs in cervical cancer. Chin. Med. J. (Engl.). 125 4270-4276 (2012)

15. Cheng, Y. et al. MicroRNA-106b is involved in transforming growth factor $\beta 1$ induced cell migration by targeting disabled homolog 2 in cervical carcinoma. J. Exp. Clin. Cancer Res. 35, 1-11 (2016).

16. Piao, J. et al. Substrate stiffness affects epithelial-mesenchymal transition of cervical cancer cells through miR-106b and its target protein DAB2. Int. J. Oncol. 50, 2033-2042 (2017).

17. Gao, D., Zhang, Y., Zhu, M., Liu, S. \& Wang, X. MiRNA expression profiles of HPVinfected patients with cervical cancer in the Uyghur population in China. PLoS ONE 11, 1-12 (2016). 
18. Liu, F. et al. MicroRNA-27b up-regulated by human papillomavirus 16 E7 promotes proliferation and suppresses apoptosis by targeting polo-like kinase2 in cervical cancer. Oncotarget 7, 19666-19679 (2016).

19. Bauer, L. et al. Expression profiling of stem cell-related genes in neoadjuvanttreated gastric cancer: a NOTCH2, GSK3B and $\beta$-catenin gene signature predicts survival. PLOS ONE 7, 1-9 (2012).

20. Espinosa, l. et al. Gene expression analysis identifies two groups of ovarian high-grade serous carcinomas with different prognosis. Mod. Pathol. 24, 846-854 (2011).

21. Grassilli, E. et al. Inhibition of GSK3B bypass drug resistance of p53-null colon carcinomas by enabling necroptosis in response to chemotherapy. Clin. Cancer Res. 19, 3820-3831 (2013).

22. Gürsel, D. B. et al. Tight regulation between cell survival and programmed cell death in GBM stem-like cells by EGFR/GSK3b/PP2A signaling. J. Neurooncol. 121, 19-29 (2015)

23. Ma, C. et al. GSK3 $\beta$ mediates the carcinogenic effect of HPV16 in cervical cancer. Sci. Rep. 5, 2-10 (2015).

24. Rath, G., Jawanjal, P., Salhan, S., Nalliah, M. \& Dhawan, I. Clinical significance of inactivated glycogen synthase kinase $3 \beta$ in HPV-associated cervical cancer: relationship with Wnt/ $\beta$-Catenin pathway activation. Am. J. Reprod. Immunol. 73, 460-478 (2015).

25. V, B. \& G, C. The angiogenic switch in carcinogenesis. Semin. Cancer Biol. 19, 329-337 (2009).

26. Chen, B., Zhang, C., Dong, P., Guo, Y. \& Mu, N. Molecular regulation of cervical cancer growth and invasion by VEGFa. Tumor Biol. 35, 11587-11593 (2014).

27. Dibbens, J. A. et al. Hypoxic regulation of vascular endothelial growth factor mRNA stability requires the cooperation of multiple RNA elements. Mol. Biol. Cell 10, 907-919 (1999).

28. Du, K., Gong, H. \& Gong, Z. Influence of serum VEGF levels on therapeutic outcome and diagnosis / prognostic value in patients with cervical. Cancer 15, 8793-8796 (2014)

29. Kong, D., Chen, F. \& Sima, N. Focal adhesion kinases crucially regulate TGFßinduced migration and invasion of bladder cancer cells via Src kinase and Ecadherin. Onco. Targets. Ther. 10, 1783-1792 (2017).

30. Ma, K. et al. PTK2-mediated degradation of ATG3 impedes cancer cells susceptible to DNA damage treatment. Autophagy 13, 579-591 (2017).

31. Martini, M., De Santis, M. C., Braccini, L., Gulluni, F. \& Hirsch, E. PI3K AKT signaling pathway and cancer: an updated review. Ann. Med. 46, 372-383 (2014).

32. Owonikoko, T. K. \& Khuri, F. R. Targeting the PI3K/AKT/mTOR pathway: biomarkers of success and tribulation. Am. Soc. Clin. Oncol. Educ. Book 14 (384-399 (2013).

33. Bai, C. et al. Anti-proliferative effect of RCE-4 from Reineckia carnea on human cervical cancer HeLa cells by inhibiting the PI3K/Akt/mTOR signaling pathway and NF-kB activation. Naunyn Schmiedebergs Arch. Pharmacol. 389, 573-584 (2016).

34. Liang, J. \& Slingerland, J. M. Multiple roles of the PI3K/PKB (Akt) pathway in cell cycle progression. Cell Cycle 2, 336-342 (2003).

35. Eke, I. \& Cordes, N. Focal adhesion signaling and therapy resistance in cancer. Semin. Cancer Biol. 31, 65-75 (2015).

36. Gari, H. H., DeGala, G. D., Ray, R., Lucia, M. S. \& Lambert, J. R. PRL-3 engages the focal adhesion pathway in triple-negative breast cancer cells to alter actin structure and substrate adhesion properties critical for cell migration and invasion. Cancer Lett. 380, 505-512 (2016).

37. Hao, Z. et al. MicroRNA-7 inhibits metastasis and invasion through targeting focal adhesion kinase in cervical cancer. Int. J. Clin. Exp. Med. 8, 480-487 (2015).

38. Jiang, $H$. et al. Targeting focal adhesion kinase renders pancreatic cancers responsive to checkpoint immunotherapy. Nat. Med. 22, 851-860 (2016).

39. Wahid, F., Shehzad, A., Khan, T. \& Kim, Y. Y. MicroRNAs: synthesis, mechanism, function, and recent clinical trials. Biochim. Biophys. Acta 1803, 1231-1243 (2010).

40. Orang, A. V., Safaralizadeh, R. \& Kazemzadeh-Bavili, M. Mechanisms of miRNAmediated gene regulation from common downregulation to mRNA-specific upregulation. Int. J. Genomics 2014, 1-15 (2014).
41. Catalanotto, C., Cogoni, C. \& Zardo, G. MicroRNA in control of gene expression: an overview of nuclear functions. Int. J. Mol. Sci. 17, 1712-1729 (2016).

42. Vasudevan, S., Tong, Y. \& Steitz, J. A. Switching from repression to activation: microRNAs can up-regulate translation. Science 318, 1931-1934 (2007).

43. Truesdell, S. S. et al. MicroRNA-mediated mRNA translation activation in quiescent cells and oocytes involves recruitment of a nuclear microRNP. Sci. Rep. 2, 0-11 (2012).

44. Stevens, K. When microRNAs activate translation. Nat. Methods 5, 122-123 (2008).

45. Chen, C. Y. A. \& Shyu, A. B. AU-rich elements: characterization and importance in mRNA degradation. Trends Biochem. Sci. 20, 465-470 (1995).

46. Barreau, C., Paillard, L. \& Osborne, H. B. AU-rich elements and associated factors: Are there unifying principles? Nucleic Acids Res. 33, 7138-7150 (2005).

47. Cao, H. \& Lin, R. Phosphorylation of recombinant tristetraprolin in vitro. Protein J. 27, 163-169 (2008).

48. Essafi-Benkhadir, K., Pouysségur, J. \& Pagès, G. Implication of the ERK pathway on the post-transcriptional regulation of VEGF mRNA stability. Methods Mol. Biol. 661, 451-469 (2010).

49. Kurosu, T. et al. HuR keeps an angiogenic switch on by stabilising mRNA of VEGF and COX-2 in tumour endothelium. Br. J. Cancer 104, 819-829 (2011).

50. Chang, S. H. et al. Antagonistic function of the RNA-binding protein HuR and miR-200b in post-transcriptional regulation of vascular endothelial growth factor-a expression and angiogenesis. J. Biol. Chem. 288, 4908-4921 (2013).

51. Brazma, A. et al. Minimum information about a microarray experiment (MIAME)-toward standards for microarray data. Nat. Genet. 29, 365-371 (2001).

52. Ramasamy, A., Mondry, A., Holmes, C. C. \& Altman, D. G. Key issues in conducting a meta-analysis of gene expression microarray datasets. PLoS Med. $\mathbf{5}$, 1320-1332 (2008).

53. Vasaikar, S. V., Straub, P., Wang, J. \& Zhang, B. LinkedOmics: analyzing multiomics data within and across 32 cancer types. Nucleic Acids Res. 46 D956-D963 (2018).

54. R. Core Team. R: A Language and Environment for Statistical Computing, Vol. 1 (R Foundation for Statistical Computing, 2017).

55. Schwarzer, G. meta: An R package for meta-analysis. R. News 7, 40-45 (2007)

56. Egger, M., Davey Smith, G., Schneider, M. \& Minder, C. Bias in meta-analysis detected by a simple, graphical test. BMJ 315, 629-634 (1997).

57. Ritchie, M. E. et al. Limma powers differential expression analyses for RNAsequencing and microarray studies. Nucleic Acids Res. 43, e47 (2015).

58. Dweep, H. \& Gretz, N. MiRWalk2.0: a comprehensive atlas of microRNA-target interactions. Nat. Methods 12, 697 (2015).

59. Conway, J. R., Lex, A. \& Gehlenborg, N. UpSetR: an R package for the visualization of intersecting sets and their properties. Bioinformatics 33, 2938-2940 (2017).

60. Jiao, X. et al. DAVID-WS: a stateful web service to facilitate gene/protein list analysis. Bioinformatics 28, 1805-1806 (2012).

61. Szklarczyk, D. et al. STRINGv10: protein-protein interaction networks, integrated over the tree of life. Nucleic Acids Res. 43, D447-D452 (2015).

62. Chin, C. H. et al. cytoHubba: identifying hub objects and sub-networks from complex interactome. BMC Syst. Biol. 8, S11 (2014).

63. Jia, P., Kao, C.-F., Kuo, P.-H. \& Zhao, Z. A comprehensive network and pathway analysis of candidate genes in major depressive disorder. BMC Syst. Biol. 5, S12 (2011).

64. Hu, Y., Pan, Z., Hu, Y., Zhang, L. \& Wang, J. Network and pathway-based analyses of genes associated with Parkinson's disease. Mol. Neurobiol. 54, 4452-4465 (2017).

65. Rhodes, D. R. et al. ONCOMINE: A Cancer Microarray Database and Integrated Data-Mining Platform. Neoplasia 6, 1-6 (2004).

66. Kozomara, A. \& Griffiths-Jones, S. MiRBase: annotating high confidence microRNAs using deep sequencing data. Nucleic Acids Res. 42, 68-73 (2014).

67. Rehmsmeier, M., Steffen, P., Höchsmann, M., Giegerich, R. \& Ho, M. Fast and effective prediction of microRNA / target duplexes. RNA 10, 1507-1517 (2004). 